\title{
Integrity of anterior corpus callosum is well related to language
}

$$
\text { impairment after traumatic brain injury }
$$

Anterior corpus callosum integrity and language impairment after traumatic brain injury

Hae In Lee', Minjae Cho ${ }^{1,2}$, Yoonhye $\mathrm{Na}^{1,2}$, Yu Mi Hwang ${ }^{1,2}$, and Sung-Bom Pyun ${ }^{1,2, *}$

${ }^{1}$ Department of Physical Medicine and Rehabilitation, Korea University College of Medicine,

Seoul, Republic of Korea

${ }^{2}$ Brain Convergence Research Center, Korea University, Seoul, Republic of Korea

${ }^{*}$ Corresponding author:

E-mail address: rmpyun@korea.ac.kr (S.-B. Pyun) 


\section{Abstract}

\section{Background}

The corpus callosum (CC) serves as the bridge that relays information between the two cerebral hemispheres, and is one of the most commonly injured areas after traumatic brain injury (TBI). This study was designed to investigate the association between the CC integrity and language function after TBI.

\section{Methods}

We retrospectively enrolled 30 patients with TBI who underwent diffusion tensor imaging and language function evaluation using the Western Aphasia Battery. The CC was divided into five segments (C1-C5) according to its projecting fibers using Hofer's method, and fractional anisotropy (FA) values were measured using DSI studio software. The FA values of the left arcuate fasciculus and cingulum for language function and executive function, respectively, were also evaluated. Twelve healthy controls were also enrolled to compare the FA values of these tracts.

\section{Results}

The FA values of the cingulum and left arcuate fasciculus were significantly correlated with all language scores. The FA values of the entire $\mathrm{CC}$ were significantly correlated with the fluency, repetition, and aphasia quotient scores. The FA values of the anterior $\mathrm{CC}$ segment $(\mathrm{C} 1$ and C2) significantly correlated with the aphasia quotient score; $\mathrm{C} 1$ with the fluency score; and C2 with the fluency, comprehension, and repetition scores. However, the FA values of the posterior $\mathrm{CC}$ (C3-C5) were not significantly correlated with any of the language subset scores.

\section{Conclusion}

The language function in patients with TBI is correlated with the integrity of the white matter tracts important for language and attention processes. Moreover, disruption of the $\mathrm{CC}$ is 
common after $\mathrm{TBI}$, and the anterior $\mathrm{CC}$ segment plays an important role in language impairment after TBI. Therefore, analyzing CC integrity using diffusion tensor imaging can help predict language impairment in patients with TBI.

Keywords: brain injuries, traumatic, corpus callosum, diffusion tensor imaging

\section{Introduction}

Traumatic brain injury (TBI) occurs after an external force injury to the brain, such as acceleration/deceleration, concussion, or penetration [1]. The recovery pattern is quite different from that after brain infarction or hemorrhage; thus, appropriate rehabilitation intervention is required. The causes of TBI include falls, vehicle collisions, and violence, and its incidence is currently increasing, especially in young populations; it is one of the major causes of morbidity and mortality $[1,2]$. The location and severity of TBI are related to shearing forces during acceleration, deceleration, and rotation of the head during injury; the common locations of diffuse axonal injuries include the cerebral hemisphere gray-white matter interface, subcortical white matter, corpus callosum (CC), basal ganglia, dorsal lateral aspect of brainstem, and cerebellum [3].

The CC is the largest commissural white matter bundle in the brain that links the left and right cerebral hemispheres and is one of the most vulnerable sites after TBI, probably owing to its unique midline location and the high possibility of secondary injury due to elevated intracranial pressure [4]. It is of particular interest as it has a structural role in inter-hemispheric transfer of cognitive function, memory, and motor functions [5]. It can be divided into three parts-the genu, trunk, and splenium. The genu connects the prefrontal cortex; the trunk connects the motor and sensory cortices; and the splenium connects the temporal, parietal, and 
occipital cortices between the two hemispheres. Many studies have been conducted to evaluate the relationship between the CC integrity and cognitive function [6]. Previous studies have identified a positive correlation between the Mini Mental State Examination (MMSE) score and the integrity of the splenium of the $\mathrm{CC}$, and a positive correlation between working memory performance and $\mathrm{CC}$ integrity $[7,8]$. While many studies have been conducted to determine the relationship between $\mathrm{CC}$ integrity and various cognitive measures, only a few studies have focused on the correlation between $\mathrm{CC}$ integrity and language function. Although the arcuate fasciculus (AF) remains the main pathway in language processing, the $\mathrm{CC}$ is also suggested to play a role in language function, such as language lateralization between the two hemispheres $[9,10]$.

In this study, we hypothesized that damage to the $\mathrm{CC}$ is associated with impairment of language function in patients with TBI. We investigated the association between CC integrity and language function after TBI using diffusion tensor imaging (DTI).

\section{Materials and methods}

\section{Subjects}

Patients with TBI who were admitted to the Physical Medicine and Rehabilitation department of Korea University Anam Hospital in Seoul, Korea, between January 2016 and December 2019 were enrolled. The inclusion criteria included the following: (1) mild-tomoderate TBI, Glasgow Coma Scale (GCS) score of $>8$, and Rancho Los Amigos (RLA) scale score of $>4$; (2) brain lesion confirmed on computed tomography (CT) or magnetic resonance imaging (MRI); and (3) available data on MRI DTI and language function evaluation using the Korean version of the Western Aphasia Battery (WAB). The exclusion criteria included the 
following: (1) severe cognitive, behavioral, or physical impairment impeding language function evaluation; (2) history of stroke or degenerative brain disorders, such as dementia or Parkinson's disease; and (3) persistent critical medical issues, such as ventilator use, after TBI. Finally, a total of 30 patients were eligible for the study. We also included 12 healthy control subjects as the control group for comparison of DTI parameters with the TBI group.

The study was conducted retrospectively, and the study protocol was approved by the Institutional Review Board (IRB) of Korea University Anam Hospital. (IRB No. 2019AN0399)

\section{Data acquisition}

The following data were collected: age, sex, years of education, occupation, radiologic findings (skull fracture, cerebral contusion, traumatic intracerebral hemorrhage, subdural hemorrhage, or epidural hematoma) observed on CT, injury site (frontal, temporal, parietal, occipital, or left or right), GCS score on admission, RLA scale score, MMSE score for cognitive function, and WAB scores for language function evaluation, including fluency (0$20)$, comprehension (0-10), repetition (0-10), naming (0-10), and aphasia quotient (AQ). The AQ was calculated using the summation of four subset scores multiplied by 2 , with a maximum of 100 points.

\section{DTI acquisition}

DTI was performed using a 3.0 T Prisma MRI scanner (Siemens, Erlangen, Germany). High-resolution, structural T1-weighted images of the entire brain were acquired. DTI data were acquired using a spin echo single-shot planar imaging pulse sequence. The image 
parameters were as follows: number of diffusion gradient directions $=64$, matrix $=112 \times 112$, field of view $=224 \times 224 \mathrm{~mm} 2$, voxel size $=2.0 \times 2.0 \times 2.0 \mathrm{~mm} 3$, echo time $=55 \mathrm{~ms}$, repetition time $=6500 \mathrm{~ms}, \mathrm{~b}=1000 \mathrm{~s} / \mathrm{mm} 2$, slide thickness $=2.0 \mathrm{~mm}$, flip angle $=90^{\circ}$. Correction to head movement was conducted using a rigid body transformation method (rotation and translation, six parameters).

\section{Reconstruction of the white matter tract using DTI}

Reconstruction was performed using DSI Studio (http://dsi-studio.labsolver.org). The termination criteria used for fiber tracking were as follows: fractional anisotropy (FA) value of $<0.15$ and angle change of $>70^{\circ}$. The region of interest was placed on the $\mathrm{CC}$, and it was reconstructed by selecting the region of interest using Hofer's classification [11]. The regions were as follows: region I-the most anterior segment, containing fibers to the prefrontal region, region II-containing fibers to the premotor and supplementary motor cortical areas, region IIIcontaining fibers to the primary motor cortex, region IV-containing fibers to the primary sensory fibers, and region $\mathrm{V}$-containing fibers to the parietal, temporal, and occipital cortices (Fig 1). These regions were named as $\mathrm{C} 1, \mathrm{C} 2, \mathrm{C} 3, \mathrm{C} 4$, and $\mathrm{C} 5$, respectively. In addition to the $\mathrm{CC}$, we reconstructed the $\mathrm{AF}$ and cingulum, which also influence language function and executive attention, respectively. The FA values of the entire $\mathrm{CC}$ and the five $\mathrm{CC}$ regions as well as those of the cingulum and AF were obtained.

Fig. 1. The corpus callosum is divided into five segments according to Hofer's method (A). The fibers of C1-C5 on diffusion tensor tractography are shown in different colors (B).

\section{Statistical analyses}


Statistical Package for the Social Sciences version 25.0 (IBM, Armonk, NY, USA) was used for the statistical analyses. The Shapiro-Wilk test was used to test the normality of the data. An independent t-test was used to compare the FA values between the TBI and control groups and Pearson's correlation test was performed for comparisons between the FA values of each tract and language scores evaluated using the WAB. A p-value of $<0.05$ was considered statistically significant.

\section{Results}

\section{Characteristics of the subjects}

The demographic data of the 30 patients are summarized in Table 1. Among them, 22 were men and 8 were wome; the mean age was 60.7 years. The etiology of TBI was fall or slipdown in 13 patients and motor vehicular accident or other trauma in 17 patients. The average GCS and RLA scale scores were 11.0 and 5.63, respectively, and the mean MMSE score was 12.73 points. The language score evaluated using the WAB was 63.0 for $A Q$, and the average subset scores were as follows: fluency, 12.58 of 20; comprehension, 6.63 of 10; repetition, 6.77 of 10; and naming, 5.72 of 10 . The patients underwent DTI in the subacute phase.

Table 1. Demographic and clinical characteristics of the patients with traumatic brain injury $(\mathbf{N}=\mathbf{3 0})$.

\begin{tabular}{|l|c|}
\hline \multicolumn{1}{|c|}{ Variables } & Mean (Standard deviation) \\
\hline Age (years) & $60.77(17.94)$ \\
\hline Sex (male/ female) & $22 / 8$ \\
\hline Education (years) & $10.83(3.10)$ \\
\hline Cause of TBI (fall/other) & $13 / 17$ \\
\hline
\end{tabular}




\begin{tabular}{|l|c|}
\hline Glasgow Coma Scale score (3-15) & $11.0(4.30)$ \\
\hline Rancho Los Amigos scale score(1-8) & $5.63(0.96)$ \\
\hline MMSE score & $12.73(8.37)$ \\
\hline Language function (WAB) & $12.58(4.99)$ \\
\hline Fluency (20) & $6.63(3.02)$ \\
\hline Comprehension (10) & $6.77(3.37)$ \\
\hline Repetition (10) & $5.72(3.24)$ \\
\hline Naming (10) & $63.0(27.06)$ \\
\hline Aphasia quotient (100) & \\
\hline
\end{tabular}

TBI: traumatic brain injury, MMSE: Mini-Mental State Examination, WAB: Western Aphasia Battery

\section{Comparison of the FA values between the TBI and control groups}

The control group that underwent MRI DTI consisted of 12 healthy participants ( 1 man and 11 women, mean age $42.58 \pm 15.92$ years). The FA values of the entire $\mathrm{CC}, \mathrm{C} 1-\mathrm{C} 5, \mathrm{AF}$, and cingulum were significantly lower in the TBI group than in the control group. (Table 2)

Table 2. Comparison of the FA values for the corpus callosum, arcuate fasciculus, and cingulum between the TBI and control groups

\begin{tabular}{c|c|c|c}
\hline Tract & TBI group $(\mathbf{n}=\mathbf{3 0})$ & Control group $(\mathbf{n}=\mathbf{1 2})$ & p-value \\
\hline Corpus callosum & $0.482(0.0350)$ & $0.546(0.0182)$ & $<0.001$ \\
\hline C1 & $0.416(0.0519)$ & $0.503(0.0127)$ & $<0.001$ \\
\hline C2 & $0.452(0.0586)$ & $0.529(0.1512)$ & $<0.001$ \\
\hline C3 & $0.473(0.0986)$ & $0.565(0.1550)$ & 0.003 \\
\hline C4 & $0.456(0.0963)$ & $0.542(0.1993)$ & 0.004 \\
\hline $\begin{array}{l}\text { Left } \\
\text { arcuate fasciculus }\end{array}$ & $0.499(0.0379)$ & $0.568(0.1902)$ & $<0.001$ \\
\hline
\end{tabular}




\begin{tabular}{l|l|l|l}
\hline Cingulum & $0.444(0.0233)$ & $0.446(0.0081)$ & $<0.001$ \\
\hline
\end{tabular}

Values are presented as means, with standard deviations in parentheses.

TBI: traumatic brain injury

\section{Correlation between the FA values of the $\mathrm{CC}$ and language scores}

Pearson's correlation test was used to identify the relationship between the FA values of each tract and the WAB scores in the TBI group. The correlation analysis results between the FA values of each tract and the WAB scores are provided in Table 3. The FA values of the left AF and cingulum showed a significant correlation with all the language subset scores. Compared with the values of the $\mathrm{AF}$ and cingulum, the FA values of the entire $\mathrm{CC}$ were significantly correlated with the fluency, repetition, and AQ scores. The FA values of the anterior segment of the $\mathrm{CC}(\mathrm{C} 1$ and $\mathrm{C} 2)$ were significantly correlated with the overall language function presented by the AQ score. The FA value of C1 was significantly correlated with the fluency score and that of $\mathrm{C} 2$ was significantly correlated with the fluency, comprehension, and repetition scores. However, the FA values of the posterior segment of the $\mathrm{CC}$ (C3-C5) were not significantly correlated with any of the language subset scores.

Table 3. Correlation coefficient of the FA values in relation to the language scores of patients with traumatic brain injury

Correlation coefficient ( $p$ value)

\begin{tabular}{cccccc}
\cline { 2 - 5 } Tract & Fluency & Comprehension & Repetition & Naming & $\begin{array}{c}\text { Aphasia } \\
\text { quotient }\end{array}$ \\
\hline Corpus callosum & $0.389^{*}$ & 0.340 & $0.364^{*}$ & 0.285 & $0.392^{*}$ \\
& $(0.033)$ & $(0.066)$ & $(0.048)$ & $(0.127)$ & $(0.032)$ \\
C1 & $0.402^{*}$ & 0.301 & 0.336 & 0.271 & $0.384^{*}$ \\
& $(0.028)$ & $(0.106)$ & $(0.070)$ & $(0.148)$ & $(0.036)$ \\
\hline
\end{tabular}




\begin{tabular}{cccccc}
\hline \multicolumn{1}{c}{ C2 } & $0.481 *$ & $0.366^{*}$ & $0.385^{*}$ & 0.341 & $0.454^{*}$ \\
& $(0.007)$ & $(0.046)$ & $(0.036)$ & $(0.065)$ & $(0.012)$ \\
$\mathbf{C 3}$ & 0.277 & 0.327 & 0.367 & 0.300 & 0.359 \\
\multicolumn{1}{c}{$\mathbf{C 4}$} & $(0.146)$ & $(0.083)$ & $(0.050)$ & $(0.114)$ & $(0.056)$ \\
\multicolumn{1}{c}{$\mathbf{C 5}$} & 0.198 & 0.353 & 0.355 & 0.345 & 0.323 \\
& $(0.302)$ & $(0.061)$ & $(0.059)$ & $(0.067)$ & $(0.088)$ \\
Left & 0.254 & 0.228 & 0.277 & 0.141 & 0.254 \\
arcuate fasciculus & $(0.175)$ & $(0.226)$ & $(0.139)$ & $(0.458)$ & $(0.175)$ \\
Cingulum & $0.458^{*}$ & $0.502^{*}$ & $0.364 *$ & $0.530^{*}$ & $0.550^{*}$ \\
& $(0.011)$ & $(0.005)$ & $(0.048)$ & $(0.003)$ & $(0.002)$ \\
\hline
\end{tabular}

\section{Discussion}

This study aimed to identify the correlation between $\mathrm{CC}$ integrity and language function in patients with TBI. Our analysis showed that the overall integrity of the white matter tracts of the $\mathrm{CC}, \mathrm{AF}$, and cingulum was significantly affected after TBI. The FA values of the entire $\mathrm{CC}$ and its segments (C1-C5) were considerably lower in the TBI group than in the control group. Only FA values of the anterior segment of the $\mathrm{CC}(\mathrm{C} 1$ and $\mathrm{C} 2)$ showed a significant correlation with language function.

DTI has been widely used in studies on white matter tract injuries among patients with TBI because it can detect minor abnormalities that conventional CT or MRI cannot [12]. In TBI, consistent decrease in anisotropic diffusion has been reported in the chronic phase after TBI. However, considerable debate remains if this occurs in the acute and sub-acute phases [13]. Although the $\mathrm{CC}$ is one of the predilection sites of traumatic axonal injury, it is believed to be unevenly affected after trauma $[4,5]$. In this study, the FA values of the AF, cingulum, and CC were significantly lower in the TBI group than in the control group. Further, the FA values of all segments of the CC consistently decreased, suggesting vulnerability of the CC after brain injury. 
The majority of patients with TBI do not demonstrate classic aphasia symptoms [14], and executive function deficit does not solely explain this $[14,15]$. There is no defined role of the CC in language processing; however, some studies have suggested its role in language lateralization and non-literal language processing which are important in social communication $[10,16]$. Some studies have reported cases of aphasia after CC injury; however, the precise mechanism has not been illustrated [17-19]. One proposed theory is that the equilibrium between the bilateral hemispheres changes after trauma; damage in the left hemisphere language center results in diaschisis between the bilateral cortices and the excitability in the right hemisphere language mirror area increases. Recovery requires reestablishment of excitation balance between the bilateral hemispheres and $\mathrm{CC}$, the largest pathway that connects the two hemispheres, plays a critical role in the transcallosal diaschisis in the language area so that aphasia recovery occurs $[20,21]$. Previous studies have suggested that the anterior segment of the CC shows DTI abnormalities after trauma, making it more associated with poor prognosis $[5,7,22,23]$. One study suggested a different mechanism underlying injury in the genu and splenium: FA reduction in the genu was accompanied by apparent diffusion coefficient (ADC) increase, whereas FA reduction in the splenium occurred in the absence of ADC change. This indicates that splenium damage is most likely to be irreversible, and genu damage is more reversible [5].

In this study, the FA values of the $\mathrm{CC}$ and all the segments overtly decreased in the TBI group compared to the control group. We believe that despite the fact that the integrity of all CC segments was disrupted after trauma, different segments contribute to language impairment. In our analysis, the FA values of the anterior $\mathrm{CC}$ segment $(\mathrm{C} 1$ and $\mathrm{C} 2)$ showed a significant correlation with the overall language function (AQ score). Further, the FA value of C1 was associated with the fluency score; and that of $\mathrm{C} 2$ was related to the fluency, comprehension, and repetition scores. However, the FA values of the posterior segment of the 
CC showed no significant correlation with any of the language subset scores. Our results suggest that the FA values of the anterior segment of the $\mathrm{CC}$ have a greater relationship with language impairment after TBI, especially the fluency score among all the language subset scores. One previous study has revealed that patients who had their anterior segment of the CC (genu and/or body) surgically removed demonstrated deficits in motor behavior and transfer of somesthetic information and that the anterior portion of the genu is associated with asynchronous motor coordination and planning [24, 25]. In another study, 4 out of 36 patients with callosal infarcts had decreased verbal fluency [20]. Further, the CC undergoes structural and functional reorganization of the language network after injury, which accounts for the improvement of spontaneous speech and repetition [18]. Thus, previous studies have observed that most patients with callosal injury demonstrats verbal language impairments. Taken together, it is reasonable to expect that because the anterior segment of the $\mathrm{CC}$ is most likely to be disrupted after trauma, this part plays an important role in language function after TBI.

Some limitations of this study include its design; this study was a single institutional, retrospective study. Further, the number of patients who participated in the study was small because many patients with TBI did not undergo DTI and language evaluation owing to severe cognitive behavioral symptoms and comorbidities. A large clinical trial should be conducted in the future to better define the relationship between $\mathrm{CC}$ lesions and language function. A longitudinal study that would demonstrate the long-term effects of CC integrity disruption on language function should also be performed to further understand the underlying mechanisms.

\section{Conclusion}

In conclusion, the language function in patients with TBI is correlated with the 
bioRxiv preprint doi: https://doi.org/10.1101/2020.06.12.148569; this version posted June 12, 2020. The copyright holder for this preprint (which was not certified by peer review) is the author/funder, who has granted bioRxiv a license to display the preprint in perpetuity. It is made available under aCC-BY 4.0 International license.

integrity of the white matter tracts important for language and attention processes. Moreover, disruption of the $\mathrm{CC}$ is common after TBI, and the anterior segment of the CC plays an important role in language impairment after TBI. Therefore, analyzing CC integrity using DTI can help predict language impairment in patients with TBI.

\section{Acknowledgements}

We would like to thank Editage (www.editage.co.kr) for English language editing.

\section{Funding}

This work was supported by the National Research Foundation of Korea funded by the Korean government (MSIT) (No. 2019R1A2C2003020) and a Korea University Grant. 


\section{References}

1. Tagliaferri F, Compagnone C, Korsic M, Servadei F, Kraus J. A systematic review of brain injury epidemiology in Europe. Acta Neurochir (Wien). 2006; 148:255-268. https://doi.org/10.1007/s00701-005-0651-y PMID: 16311842

2. Lu J, Marmarou A, Choi S, Maas A, Murray G, Steyerberg EW, et al. Mortality from traumatic brain injury. Acta Neurochir Suppl. 2005; 95:281-285. https://doi.org/10.1007/3-211-32318-x_58 PMID: 16463866

3. Parizel PM, Ozsarlak, Van Goethem JW, van den Hauwe L, Dillen C, Verlooy J, et al. Imaging findings in diffuse axonal injury after closed head trauma. Eur Radiol. 1998; 8:960-965. https://doi.org/10.1007/s003300050496 PMID: 9683701

4. Kim DS, Choi HJ, Yang JS, Cho YJ, Kang SH. Radiologic Determination of Corpus Callosum Injury in Patients with Mild Traumatic Brain Injury and Associated Clinical

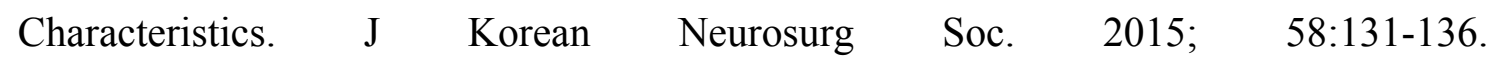
https://doi.org/10.3340/jkns.2015.58.2.131 PMID: 26361529

5. Rutgers DR, Fillard P, Paradot G, Tadié M, Lasjaunias P, Ducreux D. Diffusion tensor imaging characteristics of the corpus callosum in mild, moderate, and severe traumatic brain injury. AJNR Am J Neuroradiol. 2008; 29:1730-1735. https://doi.org/10.3174/ajnr.A1213 PMID: 18617586

6. Ljungqvist J, Nilsson D, Ljungberg M, Esbjörnsson E, Eriksson-Ritzén C, Skoglund T. Longitudinal changes in diffusion tensor imaging parameters of the corpus callosum between 6 and 12 months after diffuse axonal injury. Brain Inj. 2017; 31:344-350. https://doi.org/10.1080/02699052.2016.1256500 PMID: 28128655

7. Matsukawa H, Shinoda M, Fujii M, Takahashi O, Yamamoto D, Murakata A, et al. Genu of corpus callosum in diffuse axonal injury induces a worse 1-year outcome in patients 
with traumatic brain injury. Acta Neurochir (Wien). 2011; 153:1687-1694. https://doi.org/10.1007/s00701-011-1002-9 PMID: 21479582

8. Palacios EM, Fernandez-Espejo D, Junque C, Sanchez-Carrion R, Roig T, Tormos JM, et al. Diffusion tensor imaging differences relate to memory deficits in diffuse traumatic brain injury. BMC Neurol. 2011; 11:24. https://doi.org/10.1186/1471-2377-11-24 PMID: 21345223

9. Paul LK, Van Lancker-Sidtis D, Schieffer B, Dietrich R, Brown WS. Communicative deficits in agenesis of the corpus callosum: nonliteral language and affective prosody. Brain Lang. 2003; 85:313-324. https://doi.org/10.1016/s0093-934x(03)00062-2 PMID: 12735947

10. Hinkley LB, Marco EJ, Brown EG, Bukshpun P, Gold J, Hill S, et al. The Contribution of the Corpus Callosum to Language Lateralization. J Neurosci. 2016; 36:4522-4533. https://doi.org/10.1523/jneurosci.3850-14.2016 PMID: 27098695

11. Hofer S, Frahm J. Topography of the human corpus callosum revisited--comprehensive fiber tractography using diffusion tensor magnetic resonance imaging. Neuroimage. 2006; 32:989-994. https://doi.org/10.1016/j.neuroimage.2006.05.044 PMID: 16854598

12. Shenton ME, Hamoda HM, Schneiderman JS, Bouix S, Pasternak O, Rathi Y, et al. A review of magnetic resonance imaging and diffusion tensor imaging findings in mild traumatic brain injury. Brain Imaging Behav. 2012; 6:137-192. https://doi.org/10.1007/s11682-012-9156-5 PMID: 22438191

13. Yin B, Li DD, Huang H, Gu CH, Bai GH, Hu LX, et al. Longitudinal Changes in Diffusion Tensor Imaging Following Mild Traumatic Brain Injury and Correlation With Outcome. Front Neural Circuits. 2019; 13:28. https://doi.org/10.3389/fncir.2019.00028 PMID: 31133818

14. Vas AK, Chapman SB, Cook LG. Language impairments in traumatic brain injury: a 
window into complex cognitive performance. Handb Clin Neurol. 2015; 128:497-510. https://doi.org/10.1016/b978-0-444-63521-1.00031-5 PMID: 25701903

15. Jang SH, Lee AY, Shin SM. Injury of the Arcuate Fasciculus in the Dominant Hemisphere in Patients With Mild Traumatic Brain Injury: A Retrospective Cross-Sectional Study. Medicine (Baltimore). 2016; 95:e3007. https://doi.org/10.1097/md.0000000000003007 PMID: 26945425

16. Lábadi B, Beke AM. Mental State Understanding in Children with Agenesis of the Corpus Callosum. Front Psychol. 2017; 8:94. https://doi.org/10.3389/fpsyg.2017.00094 PMID: 28220087

17. Catani M, Jones DK, ffytche DH. Perisylvian language networks of the human brain. Ann Neurol. 2005; 57:8-16. https://doi.org/10.1002/ana.20319 PMID: 15597383

18. Yu Q, Yang W, Liu Y, Wang H, Chen Z, Yan J. Changes in the corpus callosum during the recovery of aphasia: A case report. Medicine (Baltimore). 2018; 97:e11155. https://doi.org/10.1097/md.0000000000011155 PMID: 29901647

19. Ishizaki M, Ueyama H, Nishida $Y$, Imamura S, Hirano T, Uchino M. Crossed aphasia following an infarction in the right corpus callosum. Clin Neurol Neurosurg. 2012; 114:161-165. https://doi.org/10.1016/j.clineuro.2011.09.009 PMID: 22004589

20. Saba S, Blum S. Aphasia due to isolated infarction of the corpus callosum. BMJ Case Rep. 2014; 2014 https://doi.org/10.1136/bcr-2014-204316 PMID: 24925538

21. Allendorfer JB, Storrs JM, Szaflarski JP. Changes in white matter integrity follow excitatory rTMS treatment of post-stroke aphasia. Restor Neurol Neurosci. 2012; 30:103113. https://doi.org/10.3233/rnn-2011-0627 PMID: 22233802

22. Aoki Y, Inokuchi R, Gunshin M, Yahagi N, Suwa H. Diffusion tensor imaging studies of mild traumatic brain injury: a meta-analysis. J Neurol Neurosurg Psychiatry. 2012; 83:870-876. https://doi.org/10.1136/jnnp-2012-302742 PMID: 22797288 
23. Cicuendez M, Castaño-León A, Ramos A, Hilario A, Gómez PA, Lagares A. Prognostic value of corpus callosum injuries in severe head trauma. Acta Neurochir (Wien). 2017; 159:25-32. https://doi.org/10.1007/s00701-016-3000-4 PMID: 27796652

24. Funnell MG, Corballis PM, Gazzaniga MS. Cortical and subcortical interhemispheric interactions following partial and complete callosotomy. Arch Neurol. 2000; 57:185-189. https://doi.org/10.1001/archneur.57.2.185 PMID: 10681075

25. Caillé S, Sauerwein HC, Schiavetto A, Villemure JG, Lassonde M. Sensory and motor interhemispheric integration after section of different portions of the anterior corpus callosum in nonepileptic patients. Neurosurgery. 2005; 57:50-59. https://doi.org/10.1227/01.neu.0000163089.31657.08 PMID: 15987540 
Fig. 1. The corpus callosum is divided into five segments according to Hofer's method (A). The fibers of C1-C5 on diffusion tensor tractography are shown in different colors (B).

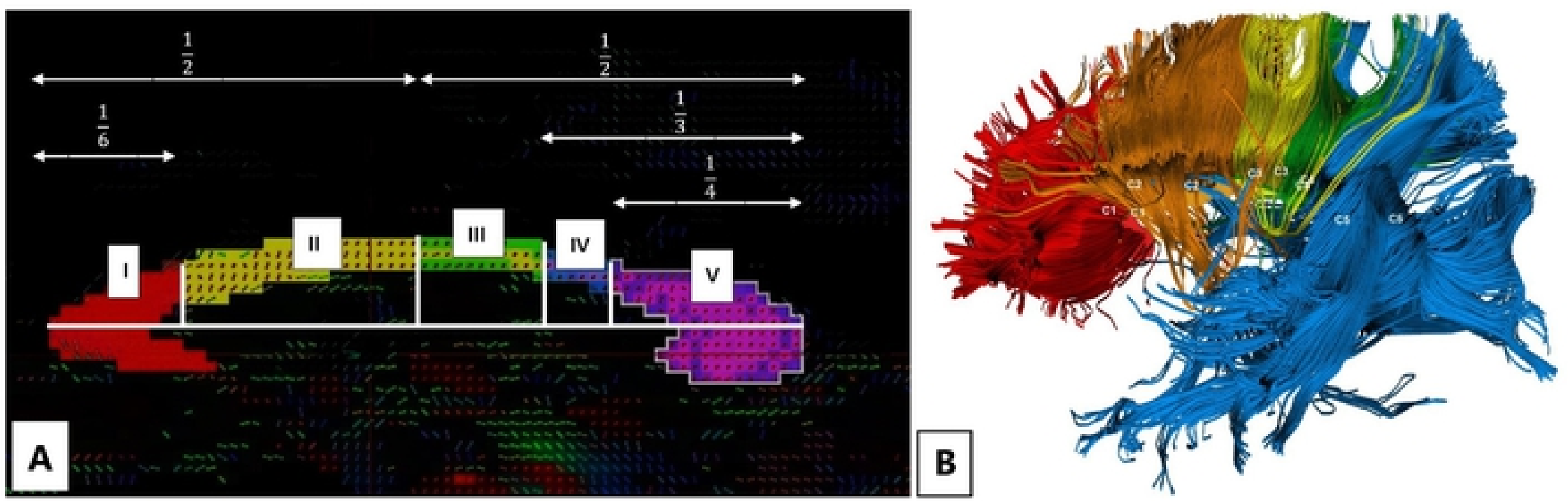

\section{Figure 1}

